\title{
Enhancing Photocatalytic Activity of ZnO Nanoparticles in a Circulating Fluidized Bed with Plasma Jets
}

\author{
Shiwei Ma ${ }^{1, \#}$, Yunyun Huang ${ }^{1, \#}$, Ruoyu Hong ${ }^{*}$, Xuesong $\mathrm{Lu}^{2}$, Jianhua $\mathrm{Li}^{3}$ \\ ${ }^{1}$ College of Chemical Engineering, Fuzhou University, Fuzhou 350108, China \\ ${ }^{2}$ School of Engineering and Physical Sciences, Heriot-Watt University, Edinburgh EH14 4AS, UK \\ ${ }^{3}$ Coal Chemical $R \&$ D Center of Kailuan Group, Tangshan 063611, China \\ ${ }^{*}$ Corresponding author. E-mail address: rhong@fzu.edu.cn (R. Hong). \\ " These authors contributed equally to this work
}

\begin{abstract}
In this work, zinc oxide ( $\mathrm{ZnO})$ nanoparticles were modified in a circulating fluidized bed through argon and hydrogen (Ar-H) alternative-current (AC) arc plasma, which shows the characteristics of non-equilibrium and equilibrium plasma at the same time. In addition, a circulating fluidized bed with two plasma jets was used for cyclic processing. The catalytic degradation performance on Rhodamine $\mathrm{B}(\mathrm{Rh} \mathrm{B})$ by $\mathrm{Ar}-\mathrm{H}$ plasma modified $\mathrm{ZnO}$ and pure $\mathrm{ZnO}$ was tested in aqueous media to identify the significant role of hydrogen atoms in $\mathrm{Rh} B$ degradation mechanism. Meanwhile, the effects of plasma treatment time on the morphology, size and photocatalytic performance of $\mathrm{ZnO}$ were also investigated. The results demonstrated that $\mathrm{ZnO}$ after 20 minutes-treatment by Ar-H plasma showed $\mathrm{Rh} B$ photocatalytic degradation rate is ten times greater than that of pure $\mathrm{ZnO}$, and the reaction follows a first-kinetics for the $\mathrm{Rh} \mathrm{B}$ degradation process. Furthermore, the photocatalyst cycle experiment curve exhibited that the modified $\mathrm{ZnO}$ still displays optimum photocatalytic activity after five cycles of experiment. The improvement of photocatalytic activity and luminescence performance attributes to the significant
\end{abstract}


increase of the surface area, and the introduction of hydrogen atoms on the surface also could enhance the time of carrier existence where the hydrogen atoms act as shallow donors.

Key words: Plasma, zinc oxide, photocatalysis, nanomaterials.

\section{Introduction}

The world environmental problems today are excessive pollution, waste of resources and energy shortages. Semiconductor photocatalysis on waste or pollution treatments is a promising environment-friendly and effective method. This technology makes full use of the semiconductor photocatalysts through photoelectric chemistry to degrade organic pollutant molecules based on the efficient use of solar energy[1-3].

Among various semiconductors, zinc oxide $(\mathrm{ZnO})$ with the wide band gap $(3.37 \mathrm{eV})[4]$, large exciton blinding energy $(60 \mathrm{meV})[5]$, good photoelectric properties, non-toxicity, abundance and environmental stability[6,7] perform much better than other photocatalysts. Therefore, $\mathrm{ZnO}$ has been widely researched for various applications including photocatalysts[8-10], chemical sensors[11], transparent electrodes[12], solar cells[13] and luminescent materials[14,15]. Especially, $\mathrm{ZnO}$ performs well on the photocatalytic. When $\mathrm{ZnO}$ is irradiated by the visible or ultraviolet light, the electrons obtain light energy to transit from the valence band to the conduction band, and generate electron-hole pairs. Simultaneously, some electrons return to the valence band in the form of heat and light emission, and other carriers move on the $\mathrm{ZnO}$ surface. Among them, electrons have strong reducibility, and photo-generated holes have strong oxidizing properties. 
They further react with pollutants to achieve photocatalytic effects[16].

However, $\mathrm{ZnO}$ nanoparticles as photocatalyst also suffers several problems: (i) The bandgap of $\mathrm{ZnO}$ is too wide for electron transition, which makes it only respond to the ultraviolet region of sunlight[8], and the ultraviolet region only occupies a ratio of 5-7\% sunlight. To expand the region of light response, modification of $\mathrm{ZnO}$ nanopowders is essential. (ii) The rapid recombination of electron-hole pairs in the catalytic process is also the main problem that seriously affects the photocatalytic performance[17-19]. To improve the photocatalytic performance of $\mathrm{ZnO}$, it needs from two aspects to increase the absorption light threshold of $\mathrm{ZnO}$ and promote the effective separation of photogenerated carriers. Increasing the specific surface area and reducing the band gap were demonstrated to be effective methods to enhance the photocatalytic ability of $\mathrm{ZnO}$. Several methods for modifying $\mathrm{ZnO}$ have been developed, such as metal element doping modification[20], non-metal element doping modification[21], semiconductor material composite loading[17,22], surface modification[23] and so on. However, these methods have disadvantages such as cumbersome preparation or serious post-treatment process or serious pollution of by-products, which limits the large-scale application of these methods[24].

Currently, plasma become a research hot point and has been extensively used in synthesis and modification of $\mathrm{ZnO}$ in recent years[25-27] because the high energy of the plasma could remove the surface state of the material, surface impurities or defects, and different plasmas would produce doping, deposition or reaction phenomena[26]. In particular, the arc plasma is highly valued due to combining the characteristics of non-equilibrium plasma and equilibrium plasma. Continuous modification of materials by arc plasma is a facile method of high yield[28].

Photocatalytic performance of $\mathrm{ZnO}$ depends on the modification conditions and methods[29]. 
Dao et al.[30]. modified $\mathrm{ZnO}$ thin films by Ar plasma and etching was observed on the surface. The grains on the surface were etched out, leading to a flatter surface with a smaller roughness. It suggested that the improvement of photoelectronic properties may be due to the effects of hydrogen ions produced by high-energy plasma ionized residual gases. Dev et al.[23]. and Baratto et al.[31]. reported similar improvements by Ar plasma treatment and proposed that the effects were attributed to incorporation of hydrogen. Nam et al.[26]. proposed the synthesis and modification methods of $\mathrm{ZnO}$ nanoparticles. No changes in specific surface area were observed, but oxygen was introduced into the $\mathrm{ZnO}$ surface, and $\mathrm{O}-\mathrm{H}$ stretching peak was increased on the $\mathrm{ZnO}$ nanoparticles surface. The increase of free radicals is the main reason to enhance the photocatalytic performance. Nitrogen $\left(\mathrm{N}_{2}\right)[32]$ and ammonia $\left(\mathrm{NH}_{3}\right)$ [33] plasma treatment were also reported and they showed similar effect of the argon plasma.

In the previous work by research groups, the preparation method of zinc oxide[34] was studied, and modification methods such as aluminum doping[35] and Ar plasma[36] were also studied. In this article, based on previous research work, we combined plasma and fluidized bed to develop a more efficient, green and convenient new modification method.

In this study, $\mathrm{ZnO}$ nanoparticles were modified by Ar and Ar-H plasma, and factors on the photocatalytic performance were studied including output voltage, plasma treatment time and plasma types. We compared the photocatalytic activity of $\mathrm{ZnO}$ before and after modification and studied the mechanism of plasma to improve the photocatalytic performance. At the same time, the role of hydrogen irons in modification process was investigated. Finally, the $\mathrm{ZnO}$ nanoparticles with excellent photocatalytic ability were successfully obtained and applied. 


\section{Materials and methods}

\subsection{Materials and apparatus}

Argon (99.99\%), hydrogen (99.99\%) were purchased from Xinhang Industrial Gases Co. Ltd. (Fuzhou, China). AC high voltage power supply and transformer were obtained from Jiaxing Jialin Electronic Technology Co. Ltd. (open circuit voltage 20 kV, Jiaxing, China). Zinc oxide(ZnO) nanoparticles were obtained from Aladdin Co. (Shanghai, China).

Fig. 1 (a) shows plasma fluidized bed equipment used in the experiments, which is made up of stainless steel. It mainly consists of two compartments: (i) The plasma generator is composed of a pair of ceramic discharge nozzles (length $150 \mathrm{~mm}$, inner diameter $40 \mathrm{~mm}$ ) and two pairs of parallel electrodes (length $120 \mathrm{~mm}$, bottom diameter $20 \mathrm{~mm}$ ); (ii) The separation and collection units include cyclone separation, bag filter and induced draft fan. The fan helps the carrier gas to circulate the $\mathrm{ZnO}$ in the plasma reactor. The products are mainly collected by a cyclone separator, and the rest is collected after being separated from the exhaust gas through a cloth bag. The details on the facility are referred to Fig. 1 (a) and Table. 1 [37].

\subsection{Modification process of $\mathrm{ZnO}$}

The modification process of $\mathrm{ZnO}$ nanoparticles by argon-hydrogen $(\mathrm{Ar}-\mathrm{H})$ arc plasma is shown in Fig. 1 (a) and the specific reaction area is shown in Fig. 1 (b). Firstly, the vacuum pump extracts the air from the reaction device to $-0.1 \mathrm{M} \mathrm{Pa}$. Then the induced draft fan was turned on to induce the Ar-H mixed gas into fluidized bed. When the AC power was supplied, the arc was generated between two parallel electrodes at the top region (as shown in the Fig. 1 (c)). The arc 
discharged in the direction of the airflow in a yellow white color. With the Ar-H gas flow rate increasing, the semicircular plasma beam enlarged and tended to be flame shape. At the same time, the semicircular plasma beam became sparse and non-uniform as the arc was elongated. The inhomogeneous arc was probably owing to the rough surfaces of the parallel electrodes. Finally, when the arc remained stable, Ar and $\mathrm{H}_{2}$ gas mixture was introduced in proportion into the plasma reactor where the ratio and the gas flow rate were controlled and adjusted by a flow meter. The plasma treatment time of $\mathrm{ZnO}$ were kept constant in five groups which were $30 \mathrm{~min}$, 60min, 90min, 120min and 150min and the corresponding products were denoted as T-1, T-2, T-3, T-4 and T-5, respectively.

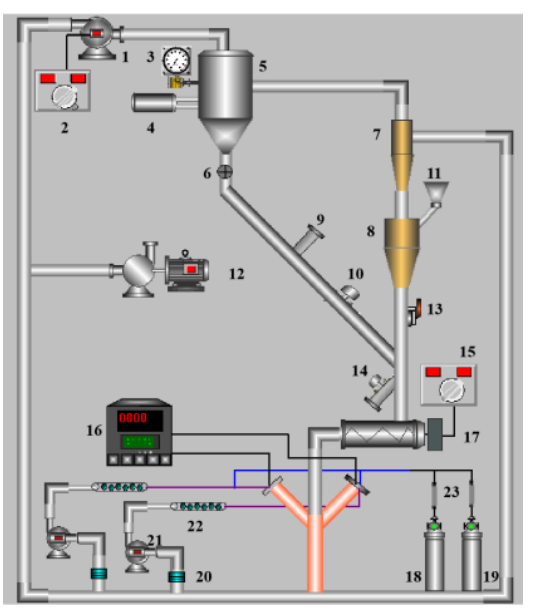

(a)

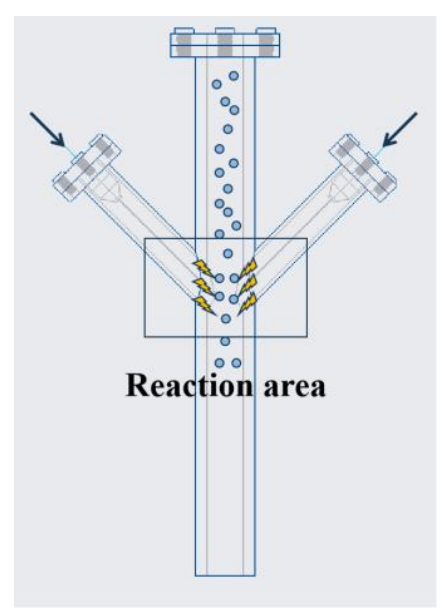

(b)

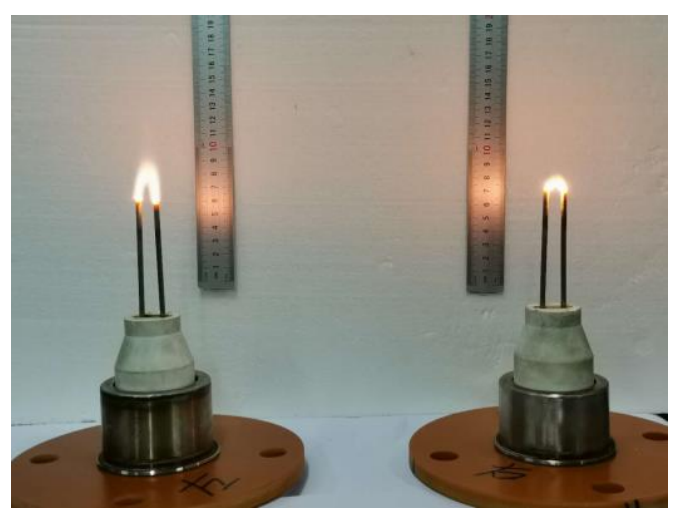

(c) 
Fig. 1. (a) Schematic diagram of modification process device, (b) specific reaction area, (c) The plane of the AC sliding arc that occurs on the top of the flat electrode.

Table. 1. Detailed parts list

\begin{tabular}{|c|c|c|c|}
\hline Part Number & Part Name & Part number & Part Name \\
\hline 1 & Induced draft fan & 12 & Vacuum pump \\
\hline 2 & Governor-1 & 13 & Valve-2 \\
\hline 3 & Pressure gauge & 14 & Outlet \\
\hline 4 & Blower & 15 & Governor-2 \\
\hline 5 & Bag separator & 16 & AC power \\
\hline 6 & Rotary feeding valve & 17 & Screw propeller \\
\hline 7 & Cyclone separator-1 & 18 & Argon \\
\hline 8 & Cyclone separator-2 & 19 & Hydrogen \\
\hline 9 & Oscillator & 20 & Filter membrane \\
\hline 10 & Valve-1 & 21 & Circulation pump \\
\hline 11 & Feeding port & 22 & Desiccant \\
\hline \multicolumn{2}{|c|}{ The diameter of all pipes in the device is $600 \mathrm{~mm}$} & 23 & Flowmeter \\
\hline
\end{tabular}

\subsection{Characterizations}

The crystal structure of $\mathrm{ZnO}$ nanoparticles was investigated by X-ray diffraction (XRD) and the data were collected on DY1602/Empyrean equipped with $\mathrm{Cu}$ Ka radiation, using a step size of $0.2^{\circ}$ and a counting time of $1 \mathrm{~s}$ per step in the range of $5-80^{\circ}$. The surface morphology of 
plasma-treated $\mathrm{ZnO}$ nanoparticles was characterized by scanning electron microscopy (SEM, Verios G4 UC). PL spectra was used to observe luminous performance at room temperature. The specific surface area was measured by Brunauer Emmett Teller (BET). To investigate the photocatalytic capacity and optical performance of $\mathrm{ZnO}$, the measurement by Ultraviolet-visble spectroscopy (UV-vis) was carried out in the range of 200-900 $\mathrm{nm}$. The band gap and the photocatalytic reaction kinetic constant are calculated by UV diffuse reflectance, absorption spectra using the Kubelka-Munk method and the degradation curve of Rhodamine B, respectively. X-ray photoelectron spectroscopy (XPS) was used to characterize element differences before and after plasma treatment.

\subsection{Measurement of photocatalytic abilities}

The photocatalytic capacity of plasma-modified $\mathrm{ZnO}$ nanoparticles was investigated by degrading Rhodamine B (Rh B). In the experiments, $0.1 \mathrm{~g}$ of the modified samples was dispersed in $200 \mathrm{~mL}$ of $\mathrm{Rh} \mathrm{B}$ aqueous solution $(10 \mathrm{mM})$. Dark treatment of suspension was kept for $60 \mathrm{~min}$ until reaching adsorption and desorption equilibrium before illumination. Then the reaction was carried out at room temperature under a $50 \mathrm{~W}$ high-pressure mercury lamp with continuous cooling water. Before the irradiation, $4 \mathrm{~mL}$ of dye mixture was taken to centrifuge for every 10 minutes, and then the UV-vis was used to analysis supernatant at $554 \mathrm{~nm}$. Therefore, the photocatalytic degradation curves of $\mathrm{Rh} \mathrm{B}$ solution were obtained, and the reaction type and kinetic constant of the degradation reaction of rhodamine B were calculated. The degradation percentages of Rh B solution were calculated using the formula $C / C_{0}$. The stability of the catalyst was also tested using cycle experiments. 


\section{Results}

3.1 XRD analysis of $\mathrm{ZnO}$ before and after modification

Fig. 2 shows the XRD patterns of $\mathrm{ZnO}$ before and after modification. The characteristic diffraction peaks of the samples are $31.7^{\circ}, 34.4^{\circ}, 36.2^{\circ}, 47.5^{\circ}, 56.7^{\circ}, 63.0^{\circ}, 66.4^{\circ}, 68.1^{\circ}$ and $69.3^{\circ}$, corresponding to the crystal planes of (100), (002), (101), (102), (110), (103), (200), (112) and (201), respectively. This result is consistent with the diffraction peaks of hexagonal wurtzite phase $\mathrm{ZnO}$ in the JCPDS standard card (No. 361451)[38]. As shown in Fig. 2, the Ar-H plasma treatment resulted in a decrease in diffraction intensity, suggesting that the plasma treatment reduced the crystallinity of $\mathrm{ZnO}$ nanoparticles and the plasma modification process did not just act on the surface.

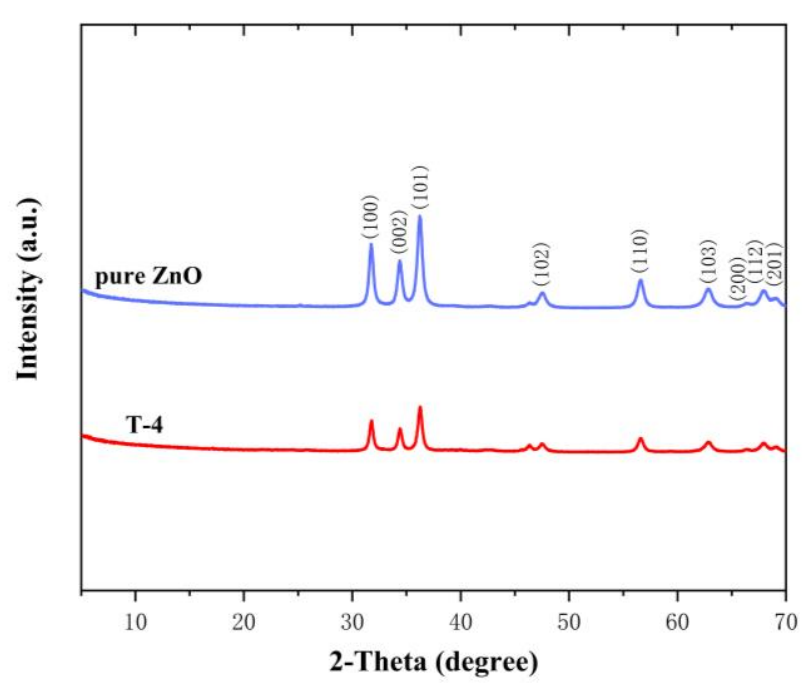

Fig. 2. XRD patterns of pure $\mathrm{ZnO}$ nanoparticles and $\mathrm{T}-4$ sample.

Fig. 3. shows (002) and (100) XRD profiles of $\mathrm{ZnO}$ nanoparticles before and after $120 \mathrm{~min}$ treatment. The (002) peak moved to a lower angle, while the (100) peak moved to a higher angle, corresponding to an increase in the lattice constant $c$ and a decrease in the lattice constant $a$, 
respectively. These results mean that new ions/atoms are introduced at the crystal plane through plasma treatment and modification, and the lattice united in the crystal grain block are stressed and deformed. At the same time, it can also be proved that plasma acts not only on the surface but also on the inside.

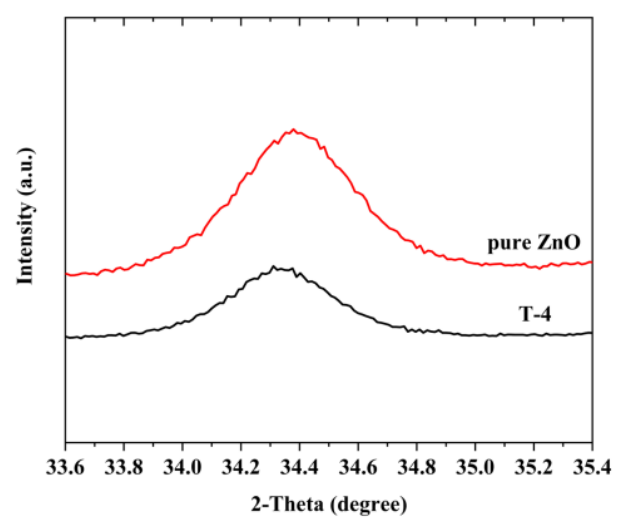

(a)

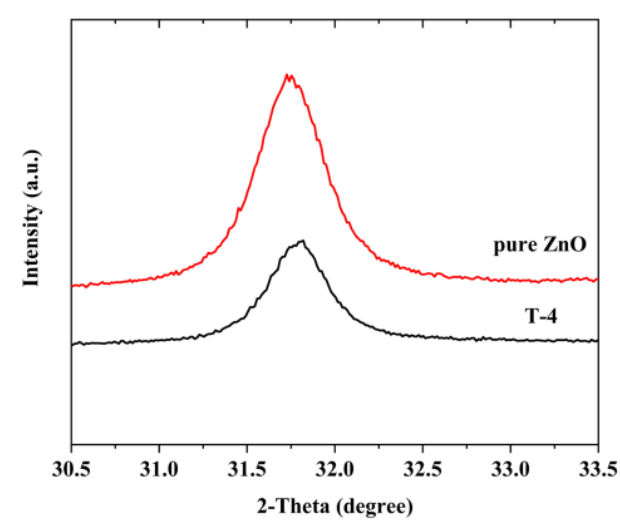

(b)

Fig. 3. Effect of the Ar-H plasma treatment time on the structural properties of the $\mathrm{ZnO}$ nanoparticles. (a) Out-of-plane 002 and (b) in-plane 100 XRD.

\subsection{SEM analysis of $\mathrm{ZnO}$ before and after modification}

SEM images of original $\mathrm{ZnO}$ and $\mathrm{T}-4$ samples are shown in Fig. 4 (a) and (b), respectively. As shown in Fig. 4 (b), the pure $\mathrm{ZnO}$ shows a spherical agglomerated structure with a diameter of 100-800 nm. After 120 minutes of modification process, the etching and bombardment effects were found on the surface of $\mathrm{ZnO}$ as well as a decrease in the degree of reunion, accompanied by smoother surfaces and smaller roughness, as shown in Fig 4 (a). In theory, the improvement of dispersibility and the reduction of nanoparticle size can effectively increase the specific surface area of $\mathrm{ZnO}$, thereby increasing the number of reactive sites and improving photocatalytic efficiency. The specific verification can be observed from the BET test below. 


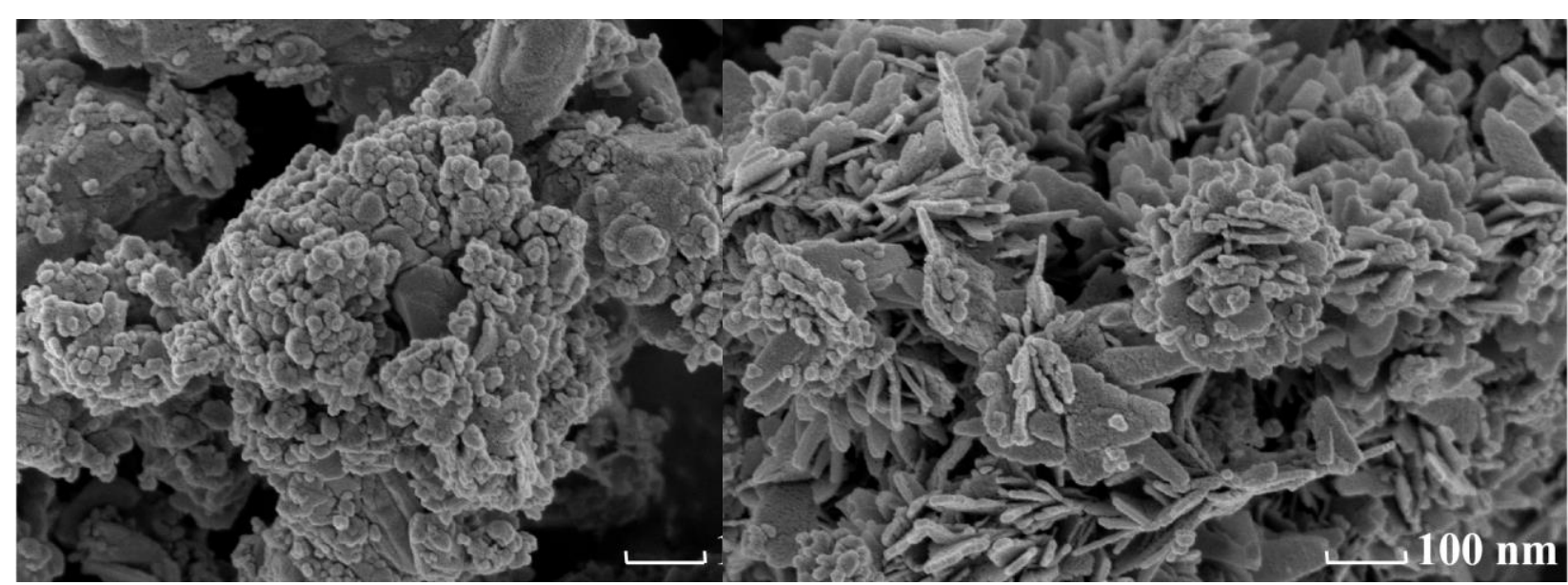

(a)

(b)

Fig. 4. SEM images of products in different time, (a) 120min plasma treatment ( $\mathrm{T}-4$ sample), (b) pure $\mathrm{ZnO}$.

3.3 Characterization of specific surface area and carrier lifetime

Fig. 5 shows the changes in specific surface area and pore size distribution before and after plasma modification of $\mathrm{ZnO}$. As shown in Fig. 5 (a), the specific surface area of $\mathrm{ZnO}$ increased to $20.5630 \mathrm{~m}^{2} / \mathrm{g}$ after 120 minutes of plasma treatment, which is 4.3 times higher than that of pure $\mathrm{ZnO}$. This may be due to the high energy of the plasma particles that etch the $\mathrm{ZnO}$ surface. The increase of the specific surface area can provide more reaction active sites for the photocatalytic reaction and improve the degradation efficiency. Fig. 5 (b) shows the pore size distribution curve of the sample. It can be seen that the small pores of pure $\mathrm{ZnO}$ and $\mathrm{T}-4$ are all distributed around 3 $\mathrm{nm}$, and the large pores are all distributed between $15-30 \mathrm{~nm}$. This porous structure is possibly caused by the gaps between small nanoparticles. 


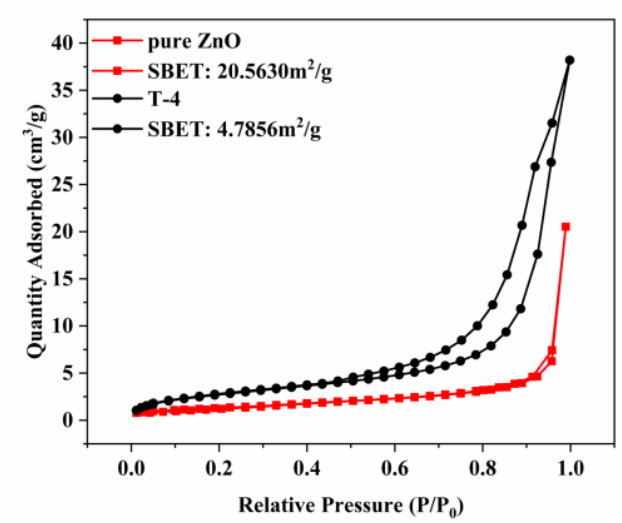

(a)

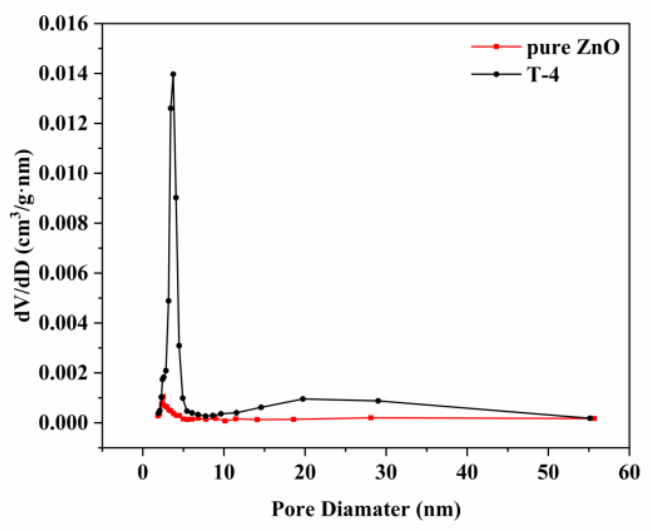

(b)

Fig. 5. (a) Nitrogen adsorption-desorption isotherms and (b) pore size distribution curves.

Photoluminescence spectrum (PL) was used to exam the luminescence intensity of $\mathrm{ZnO}$ and the recombination time of photogenerated electrons and holes. As shown in Fig. 6 (a), after plasma treatment, the luminescence intensity of $\mathrm{ZnO}$ at $380 \mathrm{~nm}$ decreased significantly, but the luminescence intensity increased at $500 \mathrm{~nm}$. This may be due to the following two reasons:

(i) The recombination rate of photogenerated electrons and holes decreases, which leads to a decrease in luminescence intensity; (ii) The slight increase in luminous intensity at $500 \mathrm{~nm}$ may be related to the increase in oxygen vacancy concentration.

The carrier lifetime was shown in Fig. 6 (b) and (c). The carrier compound time of $\mathrm{ZnO}$ was changed from $194.4 \mathrm{~ns}$ to $251.5 \mathrm{~ns}$, suggesting the photogenerated electron-hole recombination rate slowed down, which also proved the above conclusion. 


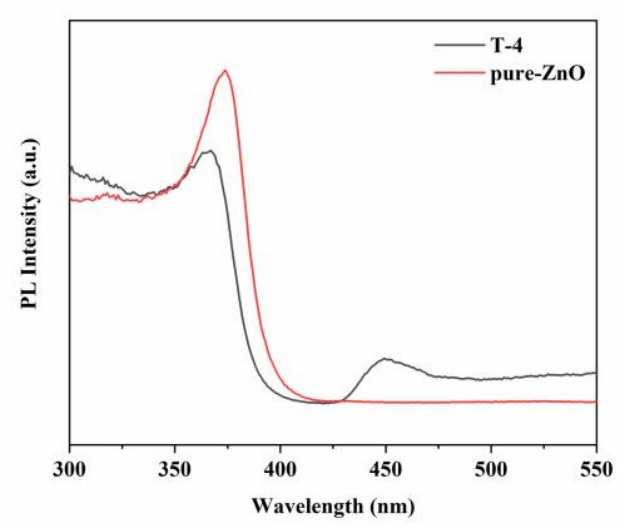

(a)

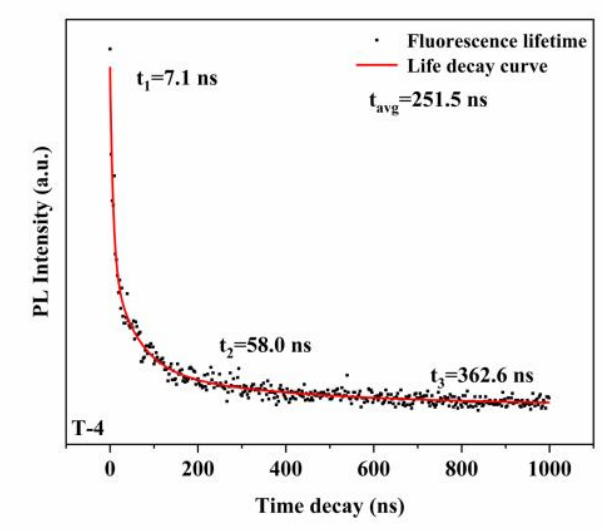

(c)

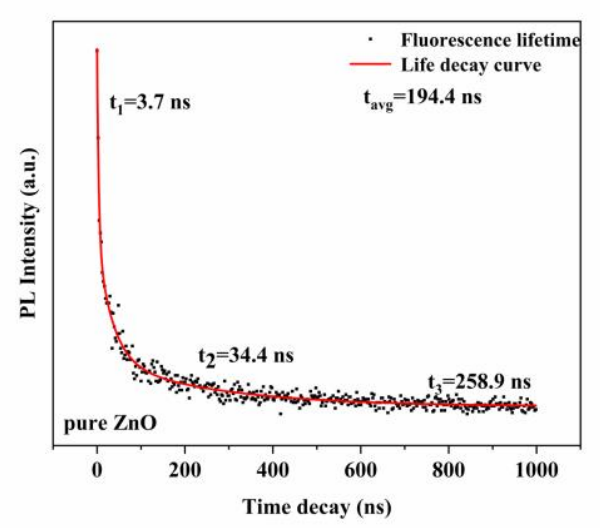

(b)

Fig. 6. (a) Photoluminescence spectra of $\mathrm{ZnO}$ and $\mathrm{T}-4$, (b) Carrier lifetime curve of pure $\mathrm{ZnO}$ and (c) Carrier lifetime curve of T-4 sample.

\subsection{XPS analysis}

X-ray electron spectroscopy (XPS) was used to evaluate the difference before and after Ar-H plasma surface treatment. Fig. 7 (a) shows the XPS spectra for Zn 2p core level peaks, the Zn $2 p$ core energy peak shifted from $1022.6 \mathrm{eV}$ to the binding energy region of $1021 \mathrm{eV}$. The results display that the $\mathrm{Zn}$ on the surface of $\mathrm{ZnO}$ changes from oxygen-rich zinc before treatment to $\mathrm{ZnO}$ containing metallic zinc after treatment. O 1s spectra is shown in Fig. 7 (b), it can be seen that the main $\mathrm{O}$ 1s peaks of $\mathrm{ZnO}$ nanoparticles are fitted to the three peaks of $531.25 \mathrm{eV}, 532.7 \mathrm{eV}$ and 
$534.4 \mathrm{eV}$, corresponding to $\mathrm{Zn}-\mathrm{O}, \mathrm{OH}$ radicals and molecular water respectively. The shoulder peaks matching the $\mathrm{OH}$ radicals and molecular water increase significantly after plasma treatment and the peak intensity increased significantly, indicating that the chemical adsorption and physical adsorption of $\mathrm{OH}$ radicals and molecular water on the surface of $\mathrm{ZnO}$ are enhanced. Fig. 7 (c) shows the specific changes in the intensity of $\mathrm{OH}$ radicals and molecular water peaks before and after plasma treatment. The results show that Ar-H plasma treatment not only increases the concentration of $\mathrm{OH}$ radicals, but also has an effect on the surface adsorption of molecular water in the air which can cleave the double bond $(\mathrm{C}=\mathrm{N})$ to decompose the $\mathrm{Rh} B$ solution and couple with the N-H single bond of the Rh B solution, improving the photocatalytic effect effectively ultimately.

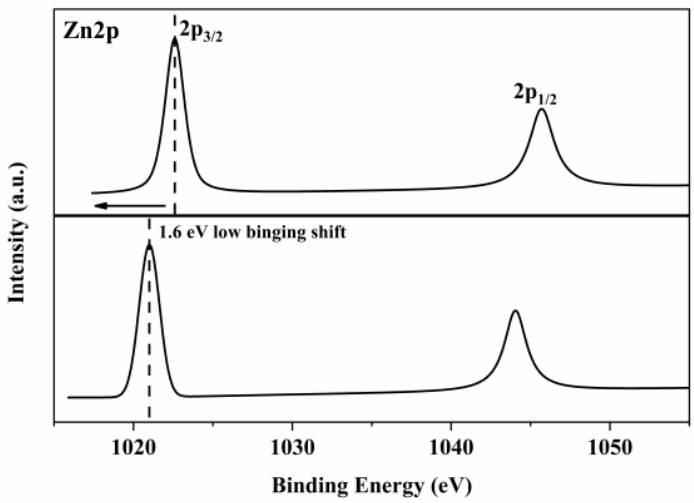

(a)

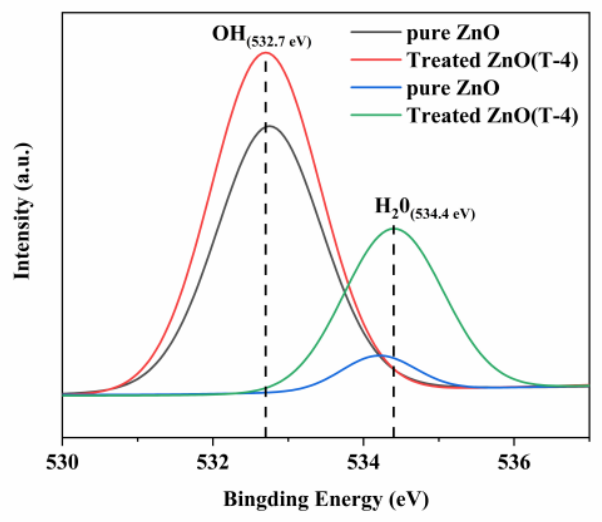

(c)

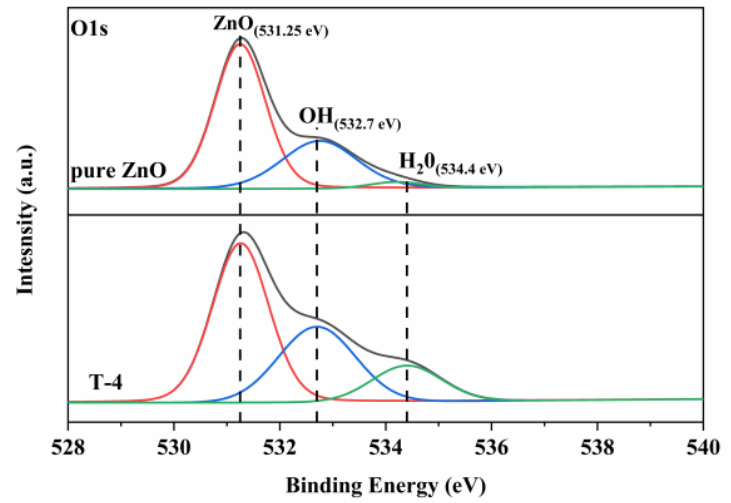

(b) 
Fig. 7. Changes in XPS spectra of $\mathrm{ZnO}$ in the (a) $\mathrm{Zn} 2 \mathrm{p}$, (b) $\mathrm{O} 1 \mathrm{~s}$ peak and (c) $\mathrm{OH}$ and $\mathrm{H}_{2} \mathrm{O}$ peaks in $\mathrm{O} 1 \mathrm{~s}$ regions before and after Ar-H plasma treatment.

\subsection{Photocatalytic degradation of Rh B solution}

To study the effect of treatment time on the activity of semiconductor catalysts, $\mathrm{ZnO}$ (T-1, $\mathrm{T}-2, \mathrm{~T}-3 . \mathrm{T}-4, \mathrm{~T}-5)$ were used for photocatalytic degradation of Rh B solution. The experimentally measured change curve of $\mathrm{C} / \mathrm{C}_{0}$ of Rh B solution with catalytic time is shown in Fig. 8 (a). The blank experiment is the Rh B solution which is directly illuminated by a $50 \mathrm{~W}$ light source with no catalyst. It is worth noting that ultraviolet light illumination in the absence of any photocatalyst or dark conditions with catalysts do not lead to the photolysis of Rh B. In fact, in the blank experiment, only $6.3 \%$ of the Rh B solution was degraded under the UV light source within 100 minutes. In the degradation experiment of adding pure $\mathrm{ZnO}$, the degradation rate of $\mathrm{Rh} \mathrm{B}$ was improved but still showed poor degradation capacity. The Ar-H plasma modified $\mathrm{ZnO}$ significantly enhanced the degradation of $\mathrm{Rh} \mathrm{B}$, and the degradation rate first increased and then decreased with time. The degradation efficiency was the highest when the plasma treatment is 120 min, and the Rh B solution was degraded by $97.8 \%$ within 40 min.

The apparent enhancement in photocatalytic degradation capacity of $\mathrm{ZnO}$ nanoparticles could be due to the effect of the increase in specific surface area (BET), decrease in electron-hole recombination rate $(\mathrm{PL})$, increase in surface $\mathrm{OH}$ radicals (XPS), and $\mathrm{H}$ atoms/ions as a shallow donor. However, long-term plasma treatment time will cause the photocatalytic ability to decrease 
(T-4), which may be attributed to the high energy of the plasma causing irreversible structural damage to the $\mathrm{ZnO}$ nanoparticles. In conclusion, the best modification time for plasma is $120 \mathrm{~min}$ and the temporal evolution about the degradation of Rh B solution over T-4 was showed in Fig. 8 (b). The concentration of $\mathrm{Rh} \mathrm{B}$ solution was indicated by the decrease in the intensity of the absorption peak.

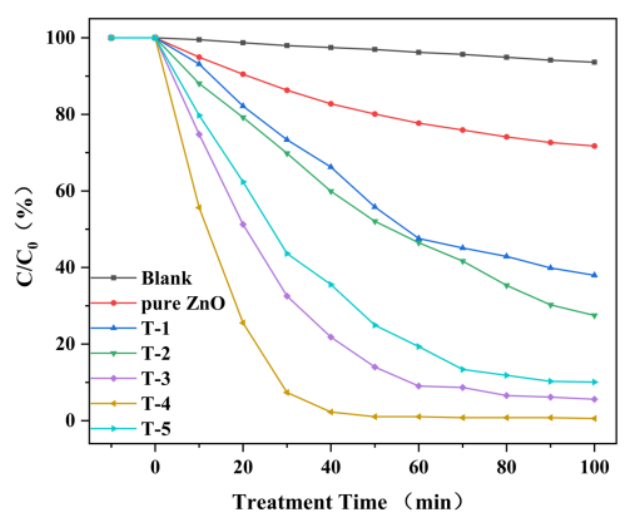

(a)

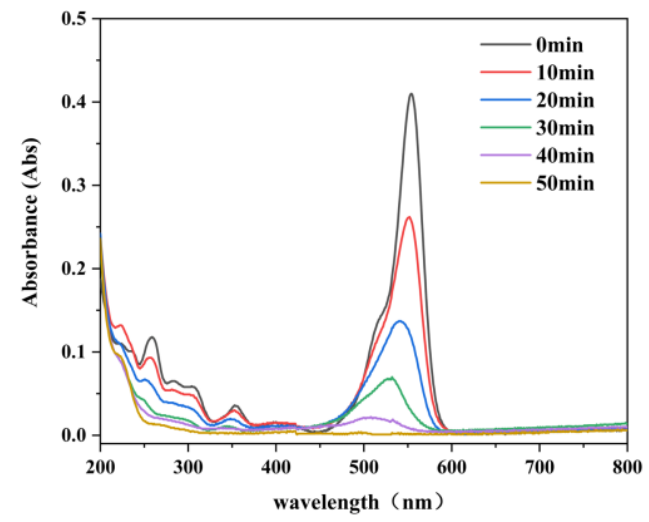

(b)

Fig. 8. (a) The Rh B concentration of different samples changes with time (b) The absorbance curve change with time when using T-4 product to degrade Rh B.

3.6 Photocatalytic degradation kinetics

The concentration of the solution can be calculated by measuring the light absorption intensity of the $\mathrm{Rh} \mathrm{B}$ solution, according to the degradation efficiency of each catalyst based on the solution concentration. It is known through equation (1) fitting that the $\mathrm{Rh} B$ degradation process conforms to the first-order reaction kinetics:

$$
\begin{aligned}
& -d C / d t=k \times C \\
& -\ln \left(C / C_{0}\right)=k \times t \\
& \ln \left(C_{0} / C\right)=k \times t
\end{aligned}
$$


where, $C, C_{0}, k, t$ represents the Rh B concentration of " $t$ " time, the initial concentration of the dye, reaction rate constant $\left(\mathrm{min}^{-1}\right)$, the time at which photo catalytic degradation takes place, respectively.

Fitting the reaction kinetics of the results of each group of photocatalytic degradation experiments, the results are shown in Fig. 9. The degradation rate of Rh B solution decreased significantly after $90 \%$ degradation, so this experiment just performed a fitting analysis on the degradation rate before $90 \%$ degradation. It can be seen from the Fig. 9 that the reaction rate constant $k$ in the blank experiment was only $0.00065 \mathrm{~min}^{-1}$. After the catalyst was added, the degradation rate of $\mathrm{Rh} \mathrm{B}$ was significantly increased, in which the reaction rate constant was $0.08933 \mathrm{~min}^{-1}$ when the treatment time was $120 \mathrm{~min}$. The fitting data of the experimental results are shown in Table 2. In all the results, $R^{2}$ is greater than 0.99 , indicating that $\ln \left(C_{0} / C\right)$ in the $\mathrm{Rh} \mathrm{B}$ degradation experiment results shows a linear relationship with time $t$, which is a first-order reaction and meet the above equation. It can be seen that the sample with the best photocatalytic degradation effect on Rh B solution is T-4.

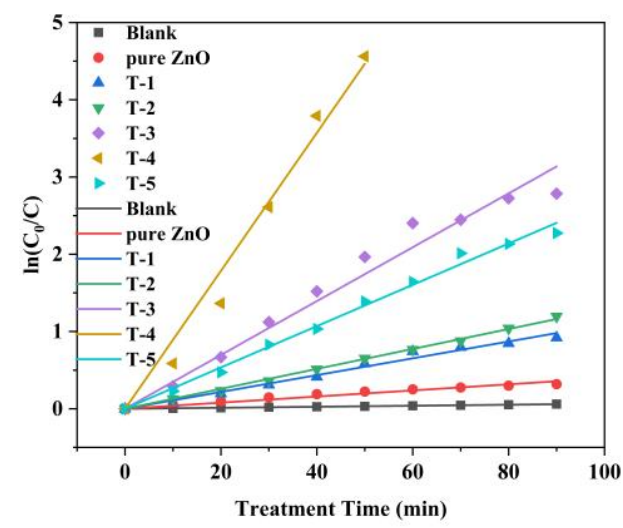

Fig. 9. The first-order reaction kinetics fitting curve of the degradation of Rh B in T4 sample.

Table 2. Degradation effect of samples with different plasma treatment time on Rh B solution 


\begin{tabular}{llll}
\hline Sample & Degradation rate $(\%)$ & Reaction rate constant $\left(\mathrm{min}^{-1}\right)$ & $R^{2}$ \\
\hline Blank & 6.4 & 0.00065 & 0.999 \\
Pure ZnO & 28.3 & 0.00396 & 0.998 \\
T-1 & 62.0 & 0.01089 & 0.994 \\
T-2 & 72.5 & 0.01291 & 0.999 \\
T-3 & 94.4 & 0.03485 & 0.991 \\
T-4 & 99.4 & 0.08933 & 0.991 \\
T-5 & 89.9 & 0.02675 & 0.997 \\
\hline
\end{tabular}

To further study the mechanism of improving photocatalysis and the mechanism of plasma modification, more characterization methods were used. The ultraviolet visible diffuse reflectance spectroscopy (DRS) was used to characterize the degree of electron-hole pairs generated by the samples and the band gap width. The ultraviolet visible (UV) absorption edge wavelength was observed at the wavelength of $375 \mathrm{~nm}$, which is the inherent absorption band gap of $\mathrm{ZnO}(3.2 \mathrm{eV})$ (shown in Fig. 10 (a)). The plasma modification process did not affect the change of the main absorption band of $\mathrm{ZnO}$, the main absorption band of $\mathrm{ZnO}$ is shorter than $400 \mathrm{~nm}$, and the absorption band of the T-4 sample was very similar to that of pure $\mathrm{ZnO}$. T-4 sample also reveals other absorption peaks, which is due to plasma treatment in the visible light region $(400-700 \mathrm{~nm})$. T-4 nanomaterial exhibits a higher absorption rate, indicating that the sample also have a certain absorption capacity of solar photons in visible light.

The energy band theory provides a theoretical basis for the catalytic process of $\mathrm{ZnO}$. As a typical semiconductor, the energy band of $\mathrm{ZnO}$ is split into two parts: the valence band and the conduction band. The valence band is usually occupied by electrons and exhibits lower energy, while the conduction band is empty and the energy is higher than the valence band. If the electron wants to transition from the valence band to the conduction band, it must pass through the band 
gap between them, expressed by $E_{g}[39]$. The electron can successfully transit from the valence band to the conduction band only if the energy provided to the electron is not less than $E_{\mathrm{g}}$.

When the energy of the incident light is not less than $\mathrm{E}_{\mathrm{g}}$, electrons will migrate from the valence band to the conduction band, and photo-generated electrons ( $\left.\mathrm{e}^{-}\right)$will be generated, and photo-generated holes $\left(\mathrm{h}^{+}\right)$will appear on the valence band. Some photogenerated electrons will consume energy to return to the valence band by luminescence or exotherm and recombine with photogenerated holes. Other photogenerated carriers are affected by the crystal defects and the surface energy state. The existence time of photogenerated carriers becomes longer, and they move to the surface of $\mathrm{ZnO}$. The photogenerated electrons that reach the surface of $\mathrm{ZnO}$ have extremely strong reducibility, and the photogenerated holes have extremely strong oxidizing properties. They will react with water and oxygen on the surface of $\mathrm{ZnO}$ to generate various free radicals, such as: $-\mathrm{OH},-\mathrm{O}^{2-}$, etc. The generated free radicals will further react with pollutants and break the chemical bonds in their molecules to achieve the purpose of photocatalytic degradation. A smaller band gap theoretically means a stronger photocatalytic ability[39].

According to the Kubelka-Munk function[40], the band gap $\left(\mathrm{E}_{\mathrm{g}}\right)$ is determined from the absorption spectra using equation as follows:

$$
\alpha h v=A\left(h v-E_{\mathrm{g}}\right)^{\eta}
$$

where, $\alpha, h, v, A, E \mathrm{~g}, \eta$ represents optical absorption coefficient, Planck constant, frequency of the incident photon, absorption constant for direct transition, band gap and index characterized by light absorption process, respectively. $\mathrm{ZnO}$ is a direct band gap semiconductor, so the value of $\eta$ is 1/2. The values of $\alpha$ and $A$ can be obtained according to the formula (3) and (4):

$$
\alpha=A(1-R)^{2} / 2 R
$$




$$
A=-\lg (R)
$$

where, $R$ is the reflectance. As shown in Fig. 10 (b), the band gap was calculated by Eqs. (2) (3) (4). The results showed that the band gap of $\mathrm{ZnO}$ was reduced from $3.22 \mathrm{eV}$ to $2.99 \mathrm{eV}$ after 120 min plasma modification process. Theoretically, the narrower the band gap, the easier it is to generate carriers, which has a higher photocatalytic degradation efficiency. The possible reason is that hydrogen acts as a shallow donor in $\mathrm{ZnO}$, leading to a reduced band gap[30].

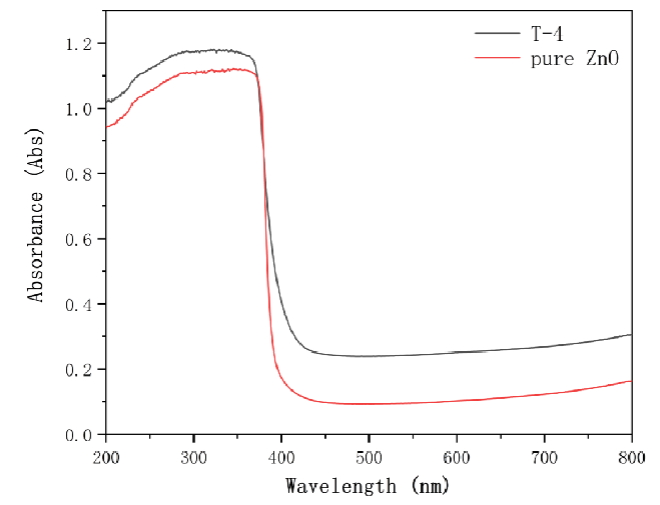

(a)

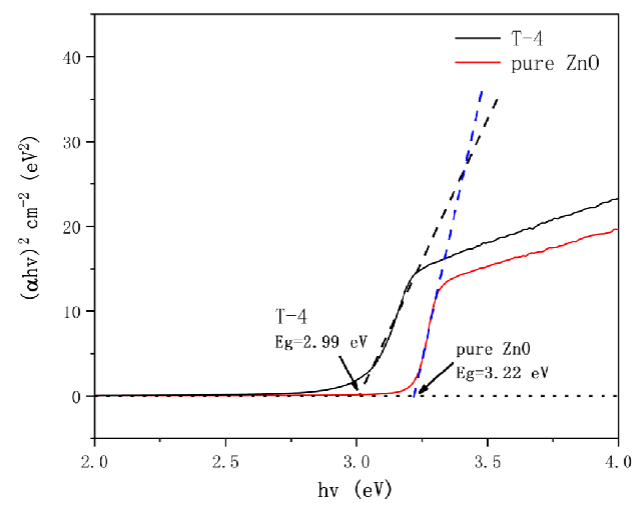

(b)

Fig. 10. (a) Ultraviolet-visible absorption spectrum and (b) the corresponding fitting curves and the fitting result of the band gap.

\subsection{Cycle experiment}

The recyling of photocatalyst is of great significance to practical applications. When evaluating the performance of a catalyst, stability is also an important indicator. The less the catalytic efficiency decreases after the catalyst is recycled, the stronger the stability. Through five repeated experiments under the same conditions, the stability of T-4 sample to the photocatalytic degradation of Rh B solution was tested. As shown in Fig. 11 (a) and (b), the T-4 sample degraded about $99.4 \%$ of $\mathrm{Rh} \mathrm{B}$ in the first circle and the degradation efficiency of $\mathrm{Rh} \mathrm{B}$ decreased to $89.76 \%$ within 100 minutes in the 5th cycle test. The reason for the decrease in photocatalytic 
efficiency may be that the hydrogen element acting as a shallow donor is separated from $\mathrm{ZnO}$ during the cycle. In addition, the process of centrifugation, washing and drying will also cause sample loss, so it is reasonable to maintain a degradation rate close to $90 \%$ after 5 cycles.

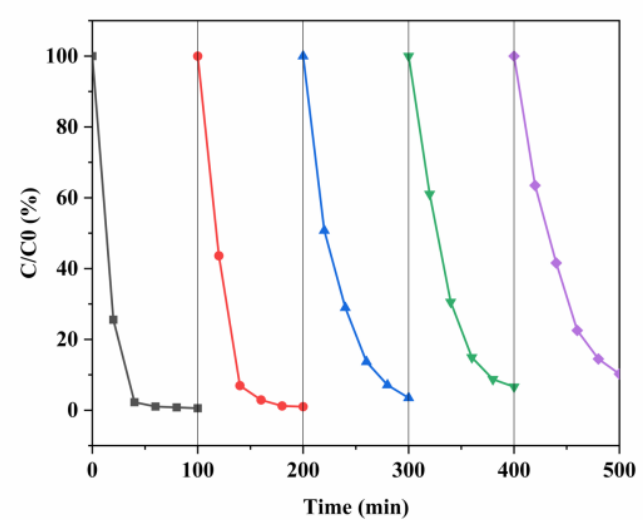

(a)

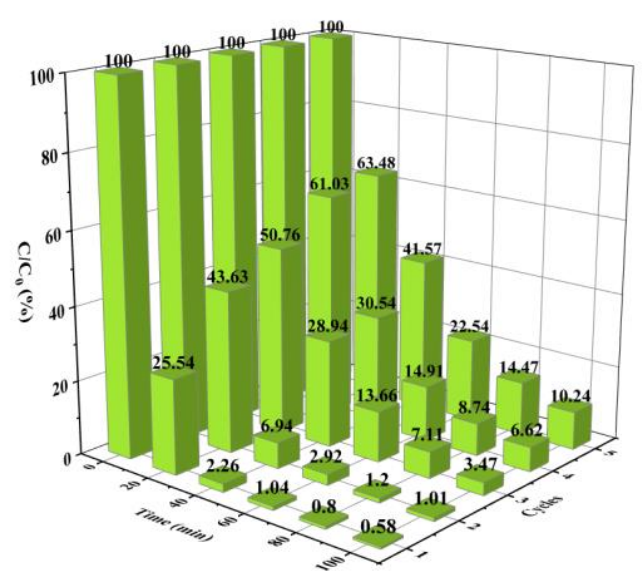

(b)

Fig. 11. (a) Cyclic degradation curve and (b) three-dimensional curve of Rh B.

\section{4. discussion}

Based on the above analysis, the mechanism to improve the photocatalytic activity was speculated. The possible mechanism responsible for the increases in photocatalytic activity is due to oxygen vacancies produced by the etching of argon-hydrogen plasma[41-43]. Oxygen vacancies are considered to be one of the main defects generated by carriers in $\mathrm{ZnO}$, and the increase in oxygen vacancies concentration will increase the photocatalytic activity of $\mathrm{ZnO}$ [44]. Firstly, the plasma particles have ultra-high energy, which can modify the surface of $\mathrm{ZnO}$ to produce some defects without changing the lattice structure. Then, hydrogen plasma can combine with oxygen to deepen the concentration of oxygen vacancies, theoretically. Another mechanism that leads to the improvement of photocatalytic performance may be resulted from hydrogen or hydrogen 
incorporation acting as shallow donors. As shown in Fig. 12, hydrogen may also occupy interstitial positions in the $\mathrm{ZnO}$ lattice and form impurity levels, which helps to increase the carrier concentration. In addition, we also discussed the problem of photocatalytic performance degradation caused by too long plasma modification. One possible explanation is that the ionized impurities are scattered, when the modification time is extended and the concentration of hydrogen ions reach a certain value, hydrogen will help the charge scattering[45,46]. On the other hand, too long plasma modification time will damage the surface of the sample, generate unsaturated dangling bonds and capture photo-generated electrons or holes[47]. These factors ultimately reduce the number of reactive sites and the concentration of surface free radicals.

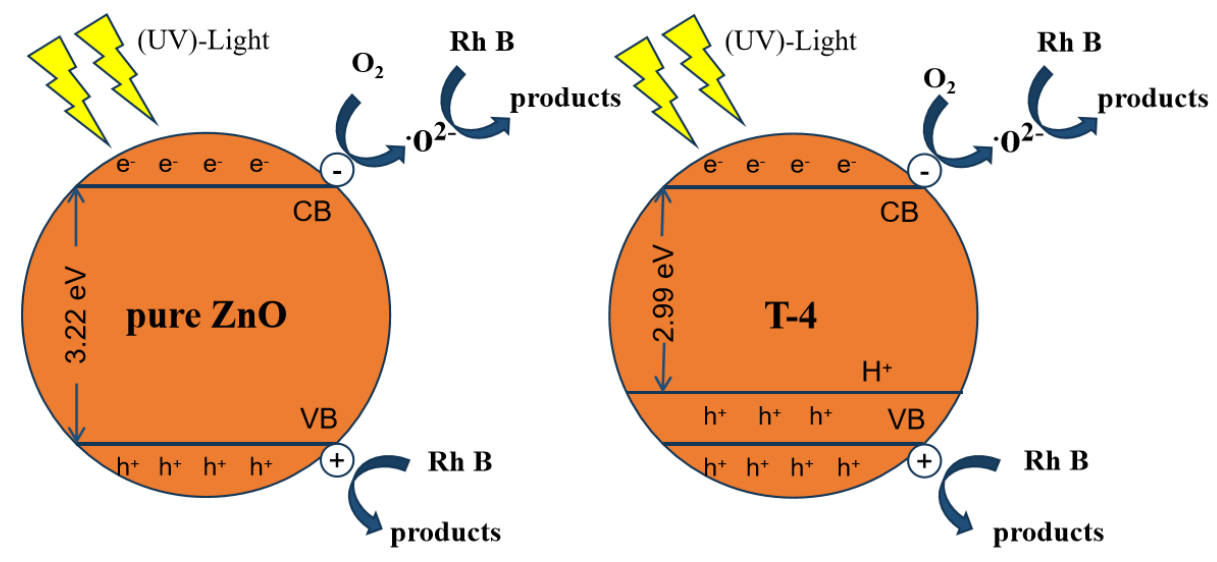

Fig. 12. Mechanism analysis of pure $\mathrm{ZnO}$ and $\mathrm{T}-4$ sample.

\section{Conclusions}

In this work, a new process combining plasma and fluidized bed has been developed for the modification of $\mathrm{ZnO}$ nanoparticles continuously using $\mathrm{AC}$ arc plasma. Compared with the traditional plasma modification methods, the process has the advantages of simple operation and continuous modification in large quantities. Under the optimal conditions of plasma modification for $120 \mathrm{~min}$, the band gap of $\mathrm{ZnO}$ was reduced to $2.99 \mathrm{eV}$, and the specific surface area was 
increased to $20.5630 \mathrm{~m}^{2} / \mathrm{g}$. As the plasma treatment time increased, the photocatalytic efficiency of the sample first increased and then decreased, and the maximum degradation rate of $\mathrm{Rh} B$ solution was $0.08933 \mathrm{~min}^{-1}$ which was 22 times than that of unmodified $\mathrm{ZnO}$. In addition, summation the characterization results of SEM, XPS, UV-vis, DRS and so on, the photocatalytic mechanism of modified $\mathrm{ZnO}$ was speculated. The Ar-H plasma treatment increased the specific surface area while reducing the band gap of $\mathrm{ZnO}$, and an impurity level was formed in the band gap of $\mathrm{ZnO}$ by hydrogen elements, which was conducive to generate and transform photo-generated electron-hole pairs. Therefore, the plasma fluidized bed modified $\mathrm{ZnO}$ nanoparticles might provide a new idea to improve the application of $\mathrm{ZnO}$ photocatalysis.

Author Contributions: Conceptualization, Shiwei. Ma. and Ruoyu. Hong.; methodology, Shiwei. Ma.; software, Shiwei. Ma.; validation, Ruoyu. Hong., Yunyun. Huang. and Jianhua. Li.; formal analysis, Shiwei. Ma.; investigation, Shiwei. Ma.; resources, Shiwei. Ma.; data curation, Shiwei. Ma.; writing—original draft preparation, Shiwei. Ma.; writing—review and editing, Jianhua. Li. and Xuesong. Li.; visualization, Shiwei. Ma.; supervision, Yunyun. Huang.; project administration, Ruoyu. Hong.; funding acquisition, Ruoyu. Hong. And Yunyun. Huang. All authors have read and agreed to the published version of the manuscript.

Acknowledgments:This research was financially supported by the National Natural Science Foundation of China (NSFC, No. 21246002), Minjiang Scholarship of Fujian Province (No. Min-Gaojiao[2010]-117), Central-government Guided Fund for Local Economic Development (No. 830170778), R\&D Fund for Strategic Emerging Industry of Fujian Province (No. 82918001), and International Cooperation Project of Fujian Science and Technology Department (No. 
830170771).

Conflicts of Interest: The authors declare no conflict of interest and all the authors approved the manuscript for publication. The funders had no role in the design of the study; in the collection, analyses, or interpretation of data; in the writing of the manuscript, or in the decision to publish the results.

\section{References}

1. Bazghale, A.A.; Mohammad-khah, A. Sonocatalytic decolorization of methylene blue from aqueous media by La:ZnO/GO nanocomposites. Res Chem Intermediat 2019, 45, 1985-2005.

2. Sun, J.B.; Gu, Y.; Lu, X.W.; Si, L.; Zhang, Q.F. Hole Doping to Enhance the Photocatalytic Activity of $\mathrm{Bi}_{4} \mathrm{NbO}_{8} \mathrm{Cl}$. Catalysts 2020, 10, 1425.

3. Reichert, G.; Hilgert, S.; Fuchs, S.; Rodrigues Azevedo, J.C. Emerging contaminants and antibiotic resistance in the different environmental matrices of Latin America. Environ Pollut 2019, 255, 113140.

4. Srikant, V.; Clarke, D.R. On the optical band gap of zinc oxide. J Appl Phys 1998, 83, 5447-5451.

5. Teke, A.; Ozgur, U.; Dogan, S.; Gu, X.; Morkoc, H.; Nemeth, B.; Nause, J.; Everitt, H.O. Excitonic fine structure and recombination dynamics in single-crystalline $\mathrm{ZnO}$. Phys Rev B 2004, 70, 195207.

6. Xu, T.; Xie, C.S. Tetrapod-like nano-particle $\mathrm{ZnO}$ /acrylic resin composite and its multi-function property. Prog Org Coat 2003, 46, 297-301.

7. Bhat, S.V.; Deepak, F.L. Tuning the bandgap of $\mathrm{ZnO}$ by substitution with $\mathrm{Mn}^{2+}, \mathrm{Co}^{2+}$ and $\mathrm{Ni}^{2+}$. Solid State Commun 2005, 135, 345-347.

8. Luna-Flores, A.; Morales, M.A.; Agustin-Serrano, R.; Portillo, R.; Luna-Lopez, J.A.; Perez-Sanchez, G.F.; Hernandez-de la Luz, A.D.; Tepale, N. Improvement of the Photocatalytic Activity of $\mathrm{ZnO} /$ Burkeite Heterostructure Prepared by Combustion Method. Catalysts 2019, 9, 817.

9. Franco, P.; Sacco, O.; De Marco, I.; Vaiano, V. Zinc Oxide Nanoparticles Obtained by Supercritical Antisolvent Precipitation for the Photocatalytic Degradation of Crystal Violet Dye. Catalysts 2019, 9, 346.

10. Al-Fori, M.; Dobretsov, S.; Myint, M.T.Z.; Dutta, J. Antifouling properties of zinc oxide nanorod coatings. Biofouling 2014, 30, 871-882.

11. Paliwal, A.; Sharma, A.; Tomar, M.; Gupta, V. Carbon monoxide (CO) optical gas sensor based on $\mathrm{ZnO}$ thin films. Sensor Actuat B-Chem 2017, 250, 679-685. 
12. Chen, S.; Wang, S. $\mathrm{ZnO}: \mathrm{H}$ indium-free transparent conductive electrodes for active-matrix display applications. Appl Phys Lett 2014, 105, 223304.

13. Rahman, M.; Wei, M.; Xie, F.; Khan, M. Efficient Dye-Sensitized Solar Cells Composed of Nanostructural ZnO Doped with Ti. Catalysts 2019, 9, 273.

14. Vanheusden, K.; Warren, W.L.; Seager, C.H.; Tallant, D.R.; Voigt, J.A.; Gnade, B.E. Mechanisms behind green photoluminescence in $\mathrm{ZnO}$ phosphor powders. J Appl Phys 1996, 79, 7983-7990.

15. Meyer, B.K.; Alves, H.; Hofmann, D.M.; Kriegseis, W.; Forster, D.; Bertram, F.; Christen, J.; Hoffmann, A.; Strassburg, M.; Dworzak, M. Bound exciton and donor-acceptor pair recombinations in ZnO. Phys Status Solidi B 2004, 241, 231-260.

16. Behnajady, M.A.; Modirshahla, N.; Hamzavi, R. Kinetic study on photocatalytic degradation of CI Acid Yellow 23 by ZnO photocatalyst. J Hazard Mater 2006, 133, 226-232.

17. Linsebigler, A.L.; Lu, G.Q.; Yates, J.T. Photocatalysis on $\mathrm{TiO}_{2}$ surfaces-principles, mechanisms, and selected results. Chem Rev 1995, 95, 735-758.

18. Han, W.; Ren, L.; Qi, X.; Liu, Y.; Wei, X.; Huang, Z.; Zhong, J. Synthesis of $\mathrm{CdS} / \mathrm{ZnO} /$ graphene composite with high-efficiency photoelectrochemical activities under solar radiation. Appl Surf Sci 2014, 299, 12-18.

19. Chen, D.; Wang, K.; Ren, T.; Ding, H.; Zhu, Y. Synthesis and characterization of the $\mathrm{ZnO} / \mathrm{mpg}-\mathrm{C}_{3} \mathrm{~N}_{4}$ heterojunction photocatalyst with enhanced visible light photoactivity. Dalton T 2014, 43, 13105-13114.

20. Kumar, P.S.; Selvakumar, M.; Bhagabati, P.; Bharathi, B.; Karuthapandian, S.; Balakumar, S. CdO/ZnO nanohybrids: facile synthesis and morphologically enhanced photocatalytic performance. Rsc Adv 2014, 4, 32977-32986.

21. Liang, P.; Zhang, C.; Sun, H.; Liu, S.; Tade, M.; Wang, S. Photocatalysis of C, N-doped $\mathrm{ZnO}$ derived from $\mathrm{ZIF}-8$ for dye degradation and water oxidation. Rsc $A d v$ 2016, 6, 95903-95909.

22. Anirudhan, T.S.; Deepa, J.R. Nano-zinc oxide incorporated graphene oxide/nanocellulose composite for the adsorption and photo catalytic degradation of ciprofloxacin hydrochloride from aqueous solutions. J Clloid Interf Sci 2017, 490, 343-356.

23. Dev, A.; Niepelt, R.; Richters, J.P.; Ronning, C.; Voss, T. Stable enhancement of near-band-edge emission of $\mathrm{ZnO}$ nanowires by hydrogen incorporation. Nanotechnology 2010, 21, 065709.

24. Qin, B.; Zhang, T.; Chen, H.; Ma, Y. The growth mechanism of few-layer graphene in the arc discharge process. Carbon 2016, 102, 494-498.

25. Chen, C.; Lu, Y.; He, H.; Xiao, M.; Wang, Z.; Chen, L.; Ye, Z. Violet Emission in ZnO Nanorods Treated with High-Energy Hydrogen Plasma. ACS Appl Mater Inter 2013, 5, 10274-10279.

26. Nam, S.H.; Boo, J.H. Enhancement of photocatalytic activity of synthesized $\mathrm{ZnO}$ nanoparticles with oxygen plasma treatment. Catal Today 2016, 265, 84-89.

27. Chen, H.; Sun, Q.; Tian, T.; Zheng, L.; Barre, M.; Monot-Laffez, I.; Makowska-Janusik, M.; Li, G.; Kassiba, A.H. Defects and microstructure of highly conducting Al-doped ZnO ceramics obtained via spark plasma sintering. J Eur Ceram Soc 2020, 40, 5529-5534.

28. Lee, D.H.; Kim, K.T.; Cha, M.S.; Song, Y.H. Optimization scheme of a rotating gliding 
arc reactor for partial oxidation of methane. P Combust Inst 2007, 31, 3343-3351.

29. Gaspar, D.; Pereira, L.; Gehrke, K.; Galler, B.; Fortunato, E.; Martins, R. High mobility hydrogenated zinc oxide thin films. Sol Energ Mat Sol C 2017, 163, 255-262.

30. Dao, H.T.; Makino, H. Enhancement in optoelectrical properties of polycrystalline $\mathrm{ZnO}$ thin films by Ar plasma. Mat Sci Semicon Proc 2019, 96, 46-52.

31. Baratto, C.; Comini, E.; Ferroni, M.; Faglia, G.; Sberveglieri, G. Plasma-induced enhancement of UV photoluminescence in $\mathrm{ZnO}$ nanowires. Crystengcomm 2013, 15, 7981-7986.

32. Rodrigues, J.; Holz, T.; Allah, R.F.; Gonzalez, D.; Ben, T.; Correia, M.R.; Monteiro, T.; Costa, F.M. Effect of $\mathrm{N}_{2}$ and $\mathrm{H}_{2}$ plasma treatments on band edge emission of $\mathrm{ZnO}$ microrods. Sci Rep-UK 2015, 5, 10783.

33. Haye, E.; Job, N.; Wang, Y.; Penninckx, S.; Stergiopoulos, V.; Tumanov, N.; Cardinal, M.; Busby, Y.; Colomer, J.F.; Su, B.L. ZnO/Carbon xerogel photocatalysts by low-pressure plasma treatment, the role of the carbon substrate and its plasma functionalization. $J$ Colloid Interf Sci 2020, 570, 312-321.

34. Zhang, P.; Hong, R.Y.; Chen, Q.; Feng, W.G.; Badami, D. Aluminum-doped zinc oxide powders: synthesis, properties and application. J Mater Sci-Mater El 2014, 25, 678-692.

35. Zhang, P.; Hong, R.Y.; Chen, Q.; Feng, W.G. On the electrical conductivity and photocatalytic activity of aluminum-doped zinc oxide. Powder Technol 2014, 253, 360-367.

36. Wang, X.; Zhang, P.; Hong, R. Preparation and application of aluminum-doped zinc oxide powders via precipitation and plasma processing method. J Appl Polym Sci 2015, 132, 215123 .

37. Wang, F.; Hong, R. Continuous preparation of structure-controlled carbon nanoparticle via arc plasma and the reinforcement of polymeric composites. Chem Eng $J$ 2017, 328, 1098-1111.

38. Omri, K.; Bettaibi, A.; Khirouni, K.; El Mir, L. The optoelectronic properties and role of $\mathrm{Cu}$ concentration on the structural and electrical properties of $\mathrm{Cu}$ doped $\mathrm{ZnO}$ nanoparticles. Physica B 2018, 537, 167-175.

39. Ye, C.; Bando, Y.; Shen, G.; Golberg, D. Thickness-dependent photocatalytic performance of $\mathrm{ZnO}$ nanoplatelets. J Phys Chem B 2006, 110, 15146-15151.

40. Sivakarthik, P.; Thangaraj, V.; Parthibavarman, M. A facile and one-pot synthesis of pure and transition metals $(\mathrm{M}=\mathrm{Co} \& \mathrm{Ni})$ doped $\mathrm{WO}_{3}$ nanoparticles for enhanced photocatalytic performance. J Mater Sci-Mater El 2017, 28, 5990-5996.

41. Kim, W.; Bang, J.-H.; Uhm, H.S.; Lee, S.H.; Park, J.S. Effects of post plasma treatment on material properties and device characteristics in indium zinc oxide thin film transistors. Thin Solid Films 2010, 519, 1573-1577.

42. Kang, J.H.; Cho, E.N.; Kim, C.E.; Lee, M.J.; Lee, S.J.; Myoung, J.M.; Yun, I. Mobility enhancement in amorphous InGaZnO thin-film transistors by Ar plasma treatment. Appl Phys Lett 2013, 102, 22-32.

43. Jeong, B.-J.; Jeong, J.K.; Song, Y.M.; Lee, H.D.; Choi, H.S.; Lee, G.W. Mobility Improvement of Amorphous Indium-gallium- zinc Oxide Thin Film Transistor by Roll-to-roll Compatible Plasma Treatment. J Semicond Tech Sci 2020, 20, 163-169.

44. Akopyan, I.K.; Labzovskaya, M.E.; Lisachenko, A.A.; Novikov, B.V.; Serov, A.Y.; Titov, 
V.V.; Filosofov, N.G. Manifestation of oxygen desorption in photoluminescence spectra of ZnO. Phys Solid State+ 2016, 58, 1767-1771.

45. Kim, W.M.; Kim, Y.H.; Kim, J.S.; Jeong, J.; Baik, Y.J.; Park, J.K.; Lee, K.S.; Seong, T.Y. Hydrogen in polycrystalline ZnO thin films. J Phys D Appl Phys 2010, 43, 365406.

46. Takahashi, I.; Hayashi, Y. Synthesis of hydrogen-doped zinc oxide transparent conductive films by RF magnetron sputtering. JPN J Appl Phys 2015, 54, 01 AD07.

47. Kim, H.R.; Lee, G.H.; Kim, D.H. Effects of hydrogen plasma treatment on the structural and electrical properties of sputter-deposited SnO2 thin films. J Phy D Appl Phys 2011, $44,185203$. 